\title{
Two Aspects of Using Hipparcos Data for Studying Multiperiodic Stellar Pulsations ${ }^{1}$
}

\author{
M. Jerzykiewicz \\ Wroctaw University Observatory, ul. Kopernika 11, 51-622 Wroctaw, \\ Poland
}

\begin{abstract}
Two aspects of using Hipparcos data for studying multiperiodic stellar pulsations involve (1) deriving frequencies of multiperiodic pulsators from Hipparcos Epoch Photometry, and (2) using Hipparcos parallaxes together with other data, such as the frequencies, frequency ratios and multicolor photometry, to identify pulsation modes. Details are examined by looking at the $\delta$ Scuti star DK Virginis. From Hipparcos Epoch Photometry, two frequencies are derived. These frequencies are then verified by showing that they also fit all available ground-based observations of the star. In addition, Hipparcos parallax and a photometric effective temperature are used to compare the position of DK Vir in the HR diagram with evolutionary tracks. The star turns out to be at the end of core-hydrogen-burning stage of its evolution or slightly beyond, with a mass of $2.2 M_{\odot}$, or somewhat smaller. The observed frequencies indicate that the two detected modes are $p_{3}$ and $p_{2}$, while the relatively large photometric amplitudes imply $\ell \lesssim 2$.
\end{abstract}

\section{Introduction}

DK Virginis $=$ HR 5005 (F1 IV) $=$ HIP 64769 was discovered to be a $\delta$ Scuti star by Danziger \& Dickens (1967) from $V$-filter observations made on a single night in February 1966. In March 1970, the star was re-observed by Valtier (1971) with a $y$ filter, also on a single night. According to Valtier (1971), no variation was detected. However, $y$-filter observations, obtained on seven nights in February, April and May 1973 at the Lowell Observatory by Jerzykiewicz (1975 - henceforth J75) confirmed the $\delta$ Scuti-type variability of the star. In addition, these observations showed a night-to-night light-range variation, indicating an interference of at least two short-period terms. Two frequencies, equal to $8.606 \mathrm{~d}^{-1}$ and $9.208 \mathrm{~d}^{-1}$, were subsequently derived by Peña, Peniche, \& González (1983 - henceforth PPG83) from $V$-filter observations, carried out on eight nights in March, April, and May 1980 at the San Pedro Mártir Observatory.

Hipparcos Epoch Photometry Annex (ESA 1997 - henceforth EPA) contains $124 \mathrm{Hp}$ magnitudes of DK Vir that span an interval of $3.2 \mathrm{yr}$. As for other stars observed with Hipparcos, these magnitudes are mean values of 9 measurements, obtained during the star's transit over the grid placed in front of the detector

\footnotetext{
${ }^{1}$ Based on data from the ESA Hipparcos astrometry satellite.
} 

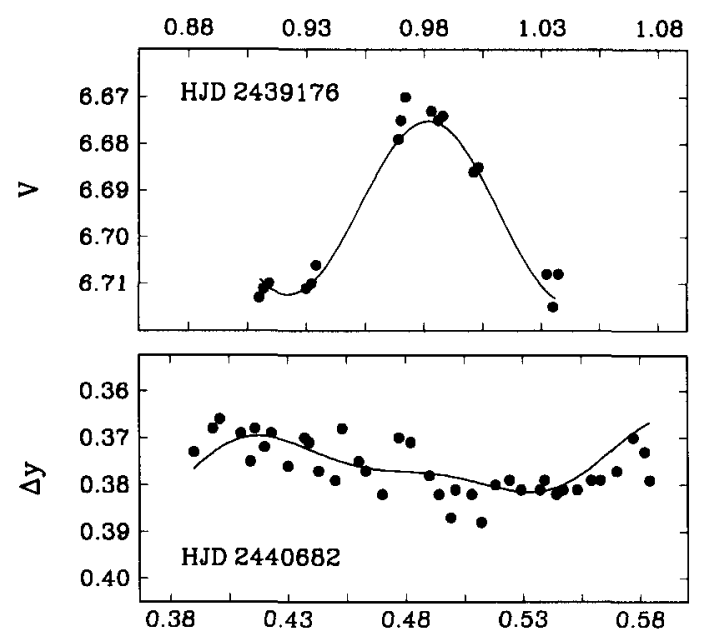

Figure 1. A comparison of the observations that were not used in the analysis with the synthetic light curves, computed from the parameters of the two-sine fit mentioned in the text. Top: The discovery night of Danziger \& Dickens (1967). Bottom: The 1970 night of Valtier (1971).

(see, e.g., Eyer et al. 1994). In addition to the magnitudes and the epochs of observations, EPA gives also the standard errors of the magnitudes, $\sigma_{H p}$, and quality flags. Two of the $124 \mathrm{Hp}$ magnitudes of DK Vir are flagged as unreliable. The remaining 122 data points were used in the analysis.

\section{The Frequencies}

In order to derive frequencies from the EPA data we slightly modified the wellknown method of least-squares frequency analysis (Lomb 1976). The modification consisted in giving each $H p$ magnitude a weight inversely proportional to $\sigma_{H p}^{2}$ and then multiplying each equation of condition by a square root of the weight.

The frequencies we obtained are equal to $f_{1}=9.2095 \mathrm{~d}^{-1}$ and $f_{2}=7.5764$ $\mathrm{d}^{-1}$. The first frequency agrees with the second frequency of PPG83 and the period given in the Hipparcos Catalogue. The second frequency is new. A twosine fit with these frequencies to the $H p$ magnitudes has a standard deviation of $3.5 \mathrm{mmag}$. The semi-amplitudes amount to $13.4 \pm 0.7 \mathrm{mmag}$ and $11.7 \pm 0.7 \mathrm{mmag}$, respectively. No further frequencies could be found. The semi-amplitudes of undetected terms are below 3.0 mmag.

The $f_{1}$ and $f_{2}$ values we derived turn out to be good enough to bridge the seven-year gap between the observations of J75 and PPG83, so that the frequencies could be refined. The refined values are equal to $9.20945 \mathrm{~d}^{-1}$ and 7.57641 $\mathrm{d}^{-1}$. The two-sine fit with these frequencies to the combined J75 and PPG83 data has a standard deviation of $5.0 \mathrm{mmag}$. This relatively large value is mainly 


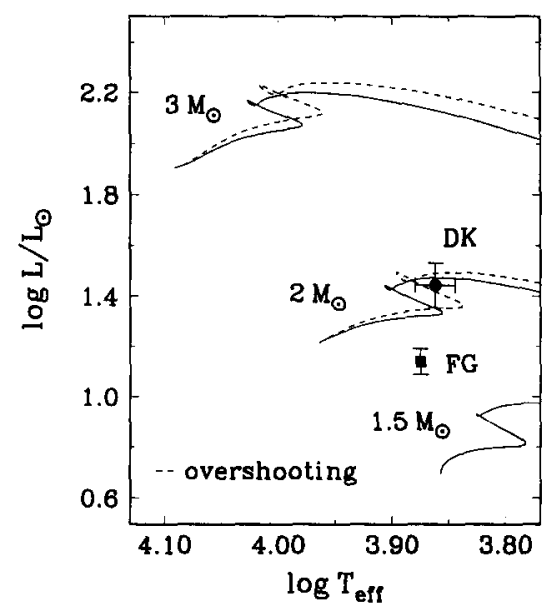

Figure 2. DK Vir (circle) and the Warsaw-New Jersey evolutionary tracks (lines) for $X=0.7$ and $Z=0.02$. The error bars extend one standard deviation from the plotted symbol. For the ordinate, the standard deviation is determined by the standard error of the parallax, while the standard deviation of the abscissa reflects the uncertainty of the $T_{\text {eff }}$ scale. FG Vir, the celebrated $\delta$ Scuti star with the largest number of identified modes (Breger et al. 1998), is also plotted.

due to the PPG83 data. Indeed, the standard deviation for these data alone is equal to $5.5 \mathrm{mmag}$, while for the $\mathrm{J} 75$ data the standard deviation amounts to $3.5 \mathrm{mmag}$. More importantly, the fit accounts also for the observations that were not used in the analysis (Fig. 1).

\section{The Mass, Evolutionary State and the Pulsation Modes}

The Hipparcos parallax yields the luminosity. This and the effective temperature, derived from the $B-V$ color index via the calibration of Code et al. (1976), defines the position of the star in the HR diagram (Fig. 2). Also shown in the figure are the Warsaw-New Jersey evolutionary tracks for $X=0.7$ and $Z=0.02$, computed with or without overshooting (dashed and solid lines, respectively), the same as those used by Breger \& Pamyatnykh (1998).

As can be seen from Fig. 2, DK Vir falls in the region of the HR diagram where the tracks for different masses intersect. Since the star's mass is not known, its evolutionary state cannot be uniquely determined. If the mass were equal to $\sim 2.2 M_{\odot}$, the star would be still in the main-sequence phase of its evolution, but decreasing the mass to $\sim 2.0 M_{\odot}$ would shift it beyond the TAMS. An investigation of the rate of change of the observed frequencies (to be done) may settle the issue and provide a better mass estimate.

Given the star's position in the temperature-luminosity plane and its mass, uncertain as it is, the observed values of the frequencies (and the ratio $f_{2} / f_{1}=$ 
0.8227 ) indicate that the $f_{1}$ and $f_{2}$ modes are $p_{3}$ and $p_{2}$, respectively. Moreover, the relatively large photometric amplitudes imply that the spherical harmonic degrees, $\ell$, are probably not greater than 2 . Since neither the degrees, $\ell$, nor the orders, $m$, are known, the number of all possible identifications of the two modes is 81 . Deriving the $\ell$ s from observations may reduce this number to 1 .

Acknowledgments. An IAU grant is gratefully acknowledged. We are indebted to Dr. A. A. Pamyatnykh for providing the evolutionary tracks used in Fig. 2. This work was supported by KBN grant 2 P03D 01611.

\section{References}

Breger, M., Zima, W., Handler, G., et al. 1998, A\&A, 331, 271

Breger, M. \& Pamyatnykh, A. A. 1998, A\&A, 332, 958

Code, A. D., Davis, J., Bless, R. C., \& Hanbury Brown, R. 1976, ApJ, 203, 417

Danziger, I. J. \& Dickens, R. J. 1967, ApJ, 149, 55

ESA 1997, The Hipparcos and Tycho Catalogues, ESA SP-1200

Eyer, L., Grenon, M., Falin, J.-L., Froeschlé, M., \& Mignard, F. 1994, Solar Phys., 152, 91

Jerzykiewicz, M. 1975, PASP, 87, 817

Lomb, N. R. 1976, Ap\&SS, 39, 447

Peña, J. H., Peniche, R., \& González, S. F. 1983, A\&AS, 53, 81

Valtier, J. C. 1971, Inf. Bull. Var. Stars, No. 556

\section{Discussion}

Géza Kovács: Our tests with RR Lyrae data show that $H p$ and ground-based $V$ data are not compatible (i.e. there is no simple transformation to match the two types of light curves). How does this result effect your analysis?

Mike Jerzykiewicz: The $H p$ response function extends over the Paschen continuum. There should be no problems with matching the $V$ amplitudes in cases when there is no wavelength-amplitude and wavelength-phase variation. 